\title{
Shear banding in molecular dynamics of polymer melts
}

Article

Published Version

Cao, J. and Likhtman, A. E. (2012) Shear banding in molecular dynamics of polymer melts. Physical Review Letters, 108 (2). 028302. ISSN 0031-9007 doi:

https://doi.org/10.1103/PhysRevLett.108.028302 Available at https://centaur.reading.ac.uk/29426/

It is advisable to refer to the publisher's version if you intend to cite from the work. See Guidance on citing.

Published version at: http://dx.doi.org/10.1103/PhysRevLett.108.028302

To link to this article DOI: http://dx.doi.org/10.1103/PhysRevLett.108.028302

Publisher: American Physical Society

All outputs in CentAUR are protected by Intellectual Property Rights law, including copyright law. Copyright and IPR is retained by the creators or other copyright holders. Terms and conditions for use of this material are defined in the End User Agreement.

\section{www.reading.ac.uk/centaur}

\section{CentAUR}

Central Archive at the University of Reading

Reading's research outputs online 


\title{
Shear Banding in Molecular Dynamics of Polymer Melts
}

\author{
Jing Cao and Alexei E. Likhtman \\ School of Mathematical and Physical Sciences, University of Reading, Reading RG6 6AX, United Kingdom
}

(Received 29 September 2011; published 11 January 2012)

\begin{abstract}
In order to establish constitutive equations for a viscoelastic fluid uniform shear flow is usually required. However, in the last 10 years S. Q. Wang and co-workers have demonstrated that some entangled polymers do not flow with the uniform shear rate as usually assumed, but instead choose to separate into fast and slow flowing regions. This phenomenon, known as shear banding, causes flow instabilities and in principle invalidates all rheological measurements when it occurs. In this Letter we report the first observation of shear banding in molecular dynamics simulations of entangled polymer melts. We show that our observations are in a very good agreement with the phenomenology developed by Fielding and Olmsted. Our findings provide a simple way of validating the empirical macroscopic phenomenology of shear banding.
\end{abstract}

DOI: 10.1103/PhysRevLett.108.028302

PACS numbers: $83.80 . \mathrm{Sg}, 47.20 . \mathrm{Ft}, 47.57 . \mathrm{Ng}$

In contrast with simple fluids such as water, viscosity of complex fluids depends not only on the shear rate (so called shear thinning or thickening), but also on the whole deformation history. This phenomenon, known as viscoelasticity and studied by rheology, has attracted great deal of attention in the last 50 years. Experimental understanding of flowing entangled polymers relies on simple rheometric flows [1]. For example, in simple shear flow fluid velocity is in one direction ( say $x$ ), and depends linearly on the coordinate in the orthogonal direction: $v_{x}=\dot{\gamma} y$. In this case $\dot{\gamma}$ is called the shear rate, and this is considered to be one of the simplest rheology experiments to perform. The simple shear data are used routinely to calibrate and verify theories of polymer dynamics, such as tube theories or constitutive equations derived from them [2]. In practice, a simple shear is obtained in couette or cone-and-plate geometries (in the limit of small gap). However, generally there is no guarantee that the liquid will flow with a uniform velocity profile-sometimes it can shear band, i.e., separate into fast and slow flowing regions. Another possibility is slip, when velocity is concentrated near the boundary. Apart from the shear history, the shear banding depends purely on the liquid properties, whereas slip is also affected by the properties of the solid plates (such as its interactions with the liquid, roughness etc.). In this Letter, we shall concentrate on the shear-banding phenomena, which is also called constitutive instability.

Theoretically, it is known that shear banding always occurs if the steady-state shear stress is a decreasing function of the shear rate. A well-known and widely studied example of a shear-banding fluid is the wormlike micelles solution [3], which exhibits a variety of flow instabilities in a wide range of parameters. Such behavior is explained by almost Maxwellian relaxation of these fluids; i.e., they relax stress with a single characteristic time scale $\tau$. If the shear rate is faster than $\tau^{-1}$, the fluid becomes unstable [4]. In contrast, polymer melts and concentrated solutions have a wide range of relaxation times, and theoretically it is not clear if their constitutive curve has a negative slope within some range of shear rates. The original DoiEdwards tube theory [5] did predict a stress maximum at the shear rate around inverse reptation time $\tau_{d}^{-1}$, whereas consecutive improvements such as contour-length fluctuations and constraint release [6] broadened the relaxation spectrum, thus reducing the tendency to shear banding. In particular, convective constraint release (CCR) was identified as a mechanism which can prevent shear banding all together $[7,8]$. CCR highlights an observation that according to the tube theory, the tube contour is being continuously stretched by the flow, which leads to the chain retraction inside the tube. This retraction naturally must cause constraint release to other surrounding chains, which in turn reduces chain alignment along the flow and therefore increases the stress. We note that this scenario is slightly unusual since a new relaxation mechanism in a nonlinear regime increases the stress rather than decreasing it. It follows from the above argument that the rate of CCR will be proportional to the shear rate in the regime of complete retraction, i.e., if the shear rate does not exceed the inverse stretch relaxation time (also called the Rouse time $\left.\tau_{R}\right)$. In this regime $\tau_{d}^{-1}<\dot{\gamma}<\tau_{R}^{-1}$ CCR becomes the dominant mechanism and thus the dynamics becomes self-similar. In other words, doubling the shear rate also doubles the CCR rate, thus doubling the relaxation rate, leading to the stress independent of the shear rate. This leads to a very uncertain prediction: since the stability of the flow is marginal if the stress is exactly constant as a function of shear rate, the small correction to the theory can tilt the situation either way. The shear banding was recently observed for polymer networks [9] using a RaPiD model where the whole polymer chain was replaced by a single particle, but the model does not have enough microscopic details to explain the origin of the shear banding. 
In the area of entangled polymers, significant progress was made in the last decade notably by Wang and coworkers, who employed the particle tracking velocimetry as the main experimental technique [10,11]. This group mainly used a $10 \%$ solution of very high molecular weight polybutadiene (around $1000 \mathrm{~kg} / \mathrm{mol}$ ) in oligomeric solvent and found a wide range of experimental conditions when the shear banding is present. This includes start-up shear, stepstrain and large oscilatory strain experiments. In start-up shear, which is the focus of this Letter, Wang and coworkers found that apart from the steady-state shear banding, some liquids show transient shear banding, which disappears in the steady state. Many of Wang's results were shown to be consistent with the tube-based constitutive equations such as Rolie-Poly [12,13]. In particular, it was shown that the shear banding should be expected even if the steady-state constitutive curve is monotonically increasing, but the system has a very small shear stress gradient, or in the presence of thermal fluctuations [14]. Theoretically, the CCR models usually have an adjustable parameter which describes the effectiveness of constraint release, and depending on this parameter the constitutive equation is either monotonic or nonmonotonic. Thus, the tube theory on its own cannot resolve the issue of flow stability and needs further input from the more microscopic models.

Although the described recent developments have changed the interpretation of many rheological experiments, they did not improve our theoretical understanding of entangled polymers dynamics and their constitutive equations. In other words, the observed shear banding invalidates some experimental data, but it is consistent with the tube theories (providing the CCR parameter is adjusted). In this Letter we turn our attention to a more microscopic tool, namely, to molecular dynamics (MD) simulations, and test whether experimental and theoretical predictions can be reproduced and understood. To our knowledge, we report the first MD simulation of entangled polymers exhibiting shear banding.

We use the simplest bead-spring model $[15,16]$ consisting of purely repulsive Lennard-Jones beads connected by finitely extensible nonlinear elastic (FENE) springs, where parameters were adjusted to prevent chain crossing. To increase the number of entanglements of these chains, we introduce a harmonic repulsive potential between beads 2 monomers apart, which increases chain stiffness. This additional potential is $U_{\text {bend }}=\frac{k_{b}}{2} \sum_{i=1}^{N-1}\left(\boldsymbol{r}_{i+1}-2 \boldsymbol{r}_{i}+\right.$ $\left.\boldsymbol{r}_{i-1}\right)^{2}$, where $k_{b}$ is the strength of bending potential (we use $k_{b}=3$ in this Letter), and $\boldsymbol{r}_{i}$ are the bead positions along the chain $(i=0 \ldots N)$. The details of the model and its equilibrium properties are discussed elsewhere [17,18]. In order to impose shear, we use Lees-Edwards boundary conditions [19] combined with two different thermostats. When using the Langevin thermostat method, we impose a uniform velocity profile by using peculiar velocities of the particles in the SLLOD $[20,21]$ equations of motion

$$
\begin{gathered}
\dot{\boldsymbol{r}}_{i}=\frac{\boldsymbol{p}_{i}}{m_{i}}+\boldsymbol{r}_{i} \cdot \nabla \boldsymbol{v} \\
\dot{\boldsymbol{p}}_{i}=\boldsymbol{f}_{i}-\boldsymbol{p}_{i} \cdot \nabla \boldsymbol{v}
\end{gathered}
$$

where $\boldsymbol{r}_{i}$ and $\boldsymbol{p}_{i}$ are the position and the peculiar momentum of particle $i$ [Eq. (1) can be considered as the definition of peculiar momentum], $\boldsymbol{f}_{i}$ is the total force on particle $i$, and

$$
\boldsymbol{\nabla} \boldsymbol{v}=\left(\begin{array}{ccc}
0 & 0 & 0 \\
\dot{\gamma} & 0 & 0 \\
0 & 0 & 0
\end{array}\right)
$$

in simple shear. Based on the SLLOD term $\boldsymbol{p}_{i} \cdot \nabla \boldsymbol{v}$, a correction force due to the $y$ component of the momentum is subtracted from the $x$ component of the force. A second method involves a dissipative particle dynamics (DPD) thermostat $[22,23]$ where the friction is calculated in respect to the velocities of surrounding particles, and thus the velocity profile is allowed to evolve. The shear in this case is driven by the boundary conditions only. We expect that the Langevin thermostat simulation will provide constitutive relations (similar to theory), whereas DPD simulations may exhibit shear banding similar to experiment. We do not impose any shear stress gradient, but naturally occurring thermal fluctuations are clearly strong because the simulation box is microscopic. In this Letter we use chain length $N=150$ with 200 chains in the box. We use the simulation box with aspect ratio 2:1:1 with the larger dimension in the shear direction $x$.

As was shown in our previous papers [17,24], one has to use the stress tensor values at each time step in order to produce accurate rheology measurements. This is due to a very large noise produced by the bond vibrations. In this work we developed careful averaging algorithms which produce reliable data for transient shear stresses. An example of such data obtained using the Langevin thermostat is shown in Fig. 1 in two different representations. Figure 1(a) demonstrates that the viscosity data are consistent with linear rheology prediction (dashed line), and the shape of the overshoots is in qualitative agreement with experimental data [25]. On the other hand, the same data replotted as stress vs strain curves show that the steadystate stress for $\dot{\gamma}=10^{-4} \tau^{-1}$ is below the curves for the $\dot{\gamma}=10^{-3} \tau^{-1}, 3 \times 10^{-5} \tau^{-1}$, which suggests a decreasing constitutive curve. The stress reaches the maximum at $\gamma \approx 2$ for all shear rates except the fastest one.

Figure 2(a) shows steady-state shear stress values for a range of chain lengths and shear rates where the data points were averaged over a long trajectory in the steady state and many independent runs-indeed we see that for the longest chains $N=150$ the constitutive curve has a maximum followed by a $5 \%$ decrease, where $\eta_{0}$ is the zero shear rate viscosity and $G_{e}=0.073 \varepsilon / \sigma^{3}$ is the entanglement plateau modulus. Viscosity for $N=150$ is 

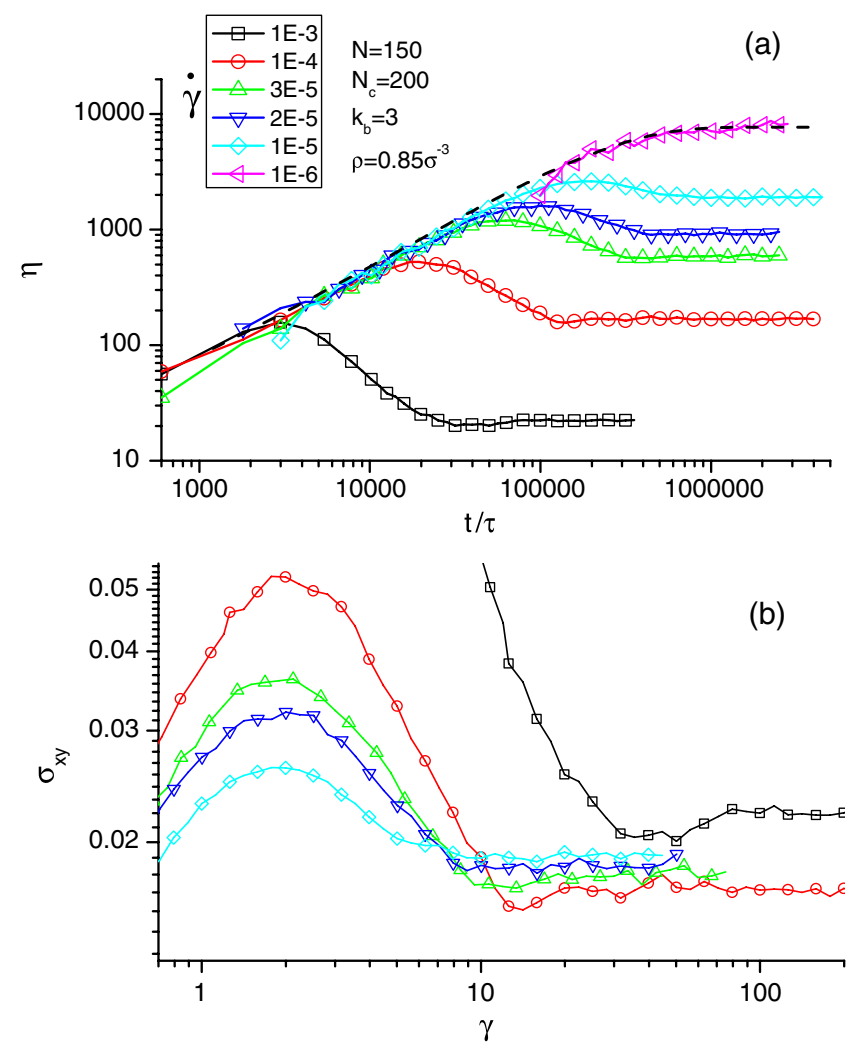

FIG. 1 (color online). (a) Viscosity as a function of time at different shear rates, the dashed line is the result from the stress relaxation function $G(t)$ in the linear viscoelastic regime, where $N$ is the monomer number of the chain, $N_{c}$ is the number of the chains in the system, $\sigma$ and $\tau$ are the Lennard-Jones length and time unit. (b) Same data as in the stress-strain representation.

$\eta_{0}=7600 \sqrt{\varepsilon m} / \sigma^{2}$ from our equilibrium simulation, where $\varepsilon=1.0 k_{B} T$ and $m$ is the mass unit. Although this decrease is quite small, it is much higher than the statistical error, which is confirmed by several independent simulations. The standard errors at minimum and maximum points of $N=150$ are around $0.2 \%$ and $0.4 \%$, respectively. We thus predict that the corresponding DPD simulation might show shear banding for the $N=150$ chains. However before discussing them we show an analogous experimental plot in Fig. 2(b) for polyisoprene melts [25]. We see a similar tendency that the slope of the constitutive curve decreases with increasing molecular weight once the shear rate exceeds inverse terminal time, approximately given by $\eta_{0} / G_{e}$. We estimate that the number of beads between entanglements in our simulation is $N_{e} \approx 15$. In the inset of Fig. 2(b) we show a comparison between simulations and experiments for the same number of entanglements. It looks like simulations show more shearthining behavior than experiments.

We now switch to the DPD simulations which allow for nonuniform velocity profiles and investigate first the shear rate $\dot{\gamma}=10^{-4} \tau^{-1}$ for chain length $N=150$ which is the minimum on the constitutive curve. Figure 3 shows the

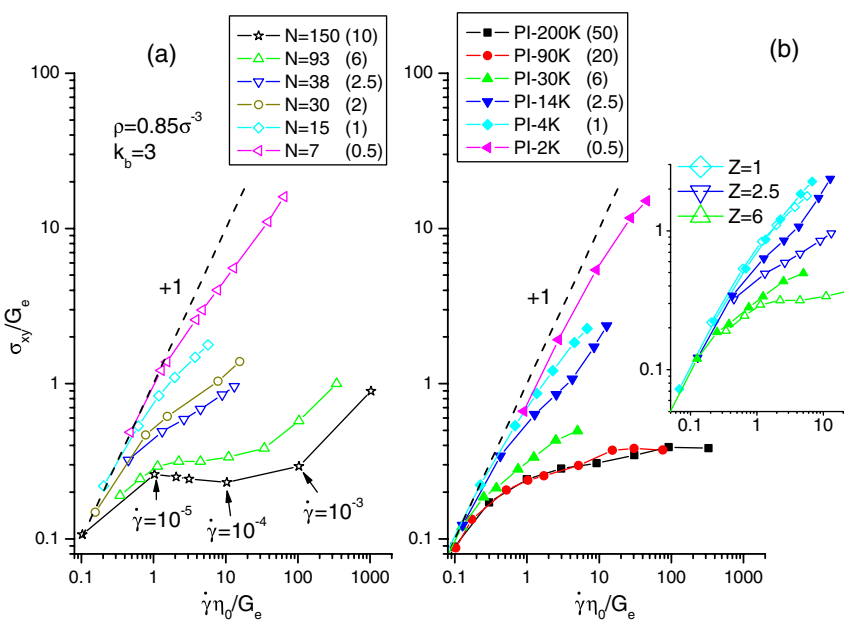

FIG. 2 (color online). Shear stress at steady state in simulations (a) and polyisoprene experiments (b) [25]. The number in the parentheses is the entanglement number $Z$ of the chain. In the inset, we compare the data between the simulations (open symbols) and the experiments (solid symbols) for the same $Z$.

measured transient stress from the two thermostats. The time step used in both DPD and Langevin simulations is $0.006 \tau$. The results agree quite well apart from the transient state where the stress in DPD simulations is slightly smaller than the one in the Langevin simulation which can be explained by the release of the linear velocity profile requirement imposed in the Langevin thermostat. The velocity profiles shown at different times in the insets clearly illustrate that the nonuniform velocity profile is responsible for this difference (different symbols in the inset indicate different independent runs). The shear bands start to develop somewhere after the stress overshoot and

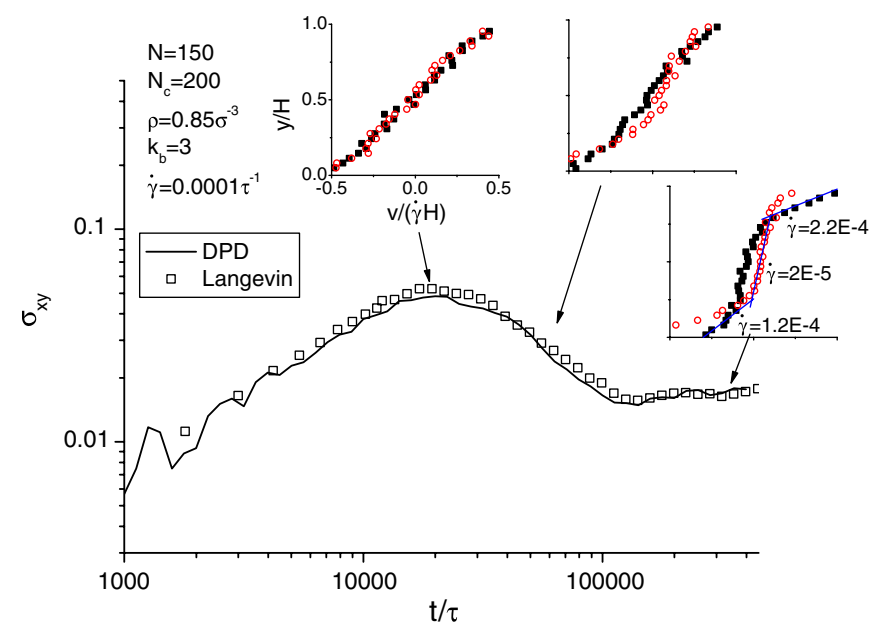

FIG. 3 (color online). Shear stress as a function of time from Langevin (open symbols) and DPD (solid line) simulations at $\dot{\gamma}=0.0001 \tau^{-1}$. The velocity profiles at different times are shown in the insets on the top, where different symbols indicate independent runs. 
fully develop in the steady state. We see that in both cases the sample had split into two bands with shear rates shown in Fig. 3 by blue (dark gray) lines. We suspect, however, that these rates are affected by the simulation box size and further investigation is needed here. We also notice that the shear bands appear in random places in the box, as illustrated by velocity profiles from the two independent runs. This is consistent with theoretical predictions [12] in the absence of stress gradients. The width of both fast and slow bands are the same in two independent runs (about half of the box). According to the theoretical picture, the width of the bands should depend on the average shear rate, which is the subject of future investigation.

It was predicted very recently [13] that the transient shear banding can appear even for shear rates on the stable branch of the constitutive curve, i.e., for $\dot{\gamma}$ such that $d \sigma / d \dot{\gamma}>0$. To test this prediction we look at velocity profiles of the DPD simulation with $\dot{\gamma}=10^{-3} \tau^{-1}$. As one can see in Fig. 4, the velocity profile becomes nonuniform after the stress maximum. The nonuniformity develops further during the stress decay, but goes away at the steady state. This is in perfect agreement with the discussion in Ref. [13] and confirms the striking fact that possibly many rheological observations are affected by the transient shear banding even at the shear rates and number of entanglement where it was not suspected. We notice, however, that the measured stresses in two simulations are quite close to each other.

In conclusion, we report the first observation of shear banding in molecular dynamics of entangled polymers. We have demonstrated that a very simple bead-spring model with slight bending potential is capable of reproducing a very rich phenomenology of shear banded states if combined with the DPD thermostat. This phenomenology is in very good agreement with the experimental observations

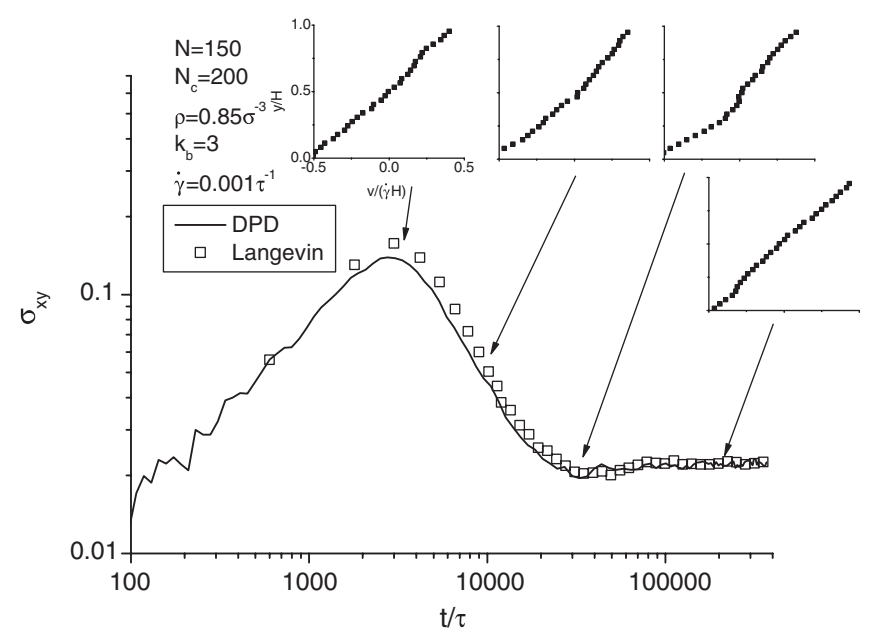

FIG. 4. Shear stress as a function of time from Langevin (open symbols) and DPD (solid line) simulations at $\dot{\gamma}=0.001 \tau^{-1}$. The velocity profiles at different times are shown in the insets. of Wang and co-workers, as well as with macroscopic calculations of Adams, Fielding, and Olmsted using the Rolie-Poly equation. We hope that this kind of MD simulation can provide a microscopic foundation and the missing ingredients of the macroscopic calculations. In particular, these calculations require a stress gradient term to be added to the Rolie-Poly equation in order to provide a unique stress-selection rule and to reproduce some other experimental observations. In the future it should be possible to investigate MD shear-banding structures for different box sizes and chain lengths in order to understand the nature of these terms and their dependence on the system parameters.

Since it is the first MD investigation of shear banding of polymer melts, many open questions remain. In particular, it seems that the shear banding in our simulations happens for a smaller number of entanglements $(Z \approx 10)$ as compared to experiments. One possible reason is the chain stiffness: our chains have less Kuhn segments per entanglement length as compared to the typical flexible polymers. Performing DPD and Langevin simulations with more flexible chains is more expensive but still possible. In fact our simulations are relatively cheap-one start-up run shown in Fig. 3 takes about 10 days on a single processor. Another direction of future investigations is the box size dependence. We note that since our boxes are microscopic (2-3 undeformed chain sizes), we can predict whether the system is stable or unstable to shear banding, but a particular shear band structure will be affected by the box size. We leave these for future publications.

[1] J.D. Ferry, Viscoelastic Properties of Polymers (Wiley, New York, 1980), Vol. 47.

[2] A.E. Likhtman and R.S. Graham, J. Non-Newtonian Fluid Mech. 114, 1 (2003).

[3] R. W. Mair and P. T. Callaghan, Europhys. Lett. 36, 719 (1996).

[4] M.E. Cates, Macromolecules 20, 2289 (1987).

[5] M. Doi and S.F. Edwards, The Theory of Polymer Dynamics. (Clarendon Press, New York, 1986).

[6] T. C. B. Mcleish, Adv. Phys. 51, 1379 (2002).

[7] G. Marrucci, J. Non-Newtonian Fluid Mech. 62, 279 (1996).

[8] A. E. Likhtman, S. T. Milner, and T. C. B. McLeish, Phys. Rev. Lett. 85, 4550 (2000).

[9] J. Sprakel, J. T. Padding, and W. J. Briels, Europhys. Lett. 93, 58003 (2011)

[10] S.-Q. Wang et al., Phys. Rev. Lett. 97, 187801 (2006).

[11] P. Tapadia, S. Ravindranath, and S.-Q. Wang, Phys. Rev. Lett. 96, 196001 (2006).

[12] P. D. Olmsted, Rheol. Acta 47, 283 (2008).

[13] J. M. Adams, S. M. Fielding, and P. D. Olmsted, J. Rheol. 55, 1007 (2011).

[14] J. M. Adams and P.D. Olmsted, Phys. Rev. Lett. 102, 067801 (2009). 
[15] M. Kroger and S. Hess, Phys. Rev. Lett. 85, 1128 (2000).

[16] K. Kremer and G. S. Grest, J. Chem. Phys. 92, 5057 (1990).

[17] A.E. Likhtman, S. K. Sukumaran, and J. Ramirez, Macromolecules 40, 6748 (2007).

[18] J. Cao and A. E. Likhtman, Phys. Rev. Lett. 104, 207801 (2010).

[19] A. W. Lees and S.F. Edwards, J. Phys. C 5, 1921 (1972).
[20] D. J. Evans and G. P. Morriss, Comput. Phys. Rep. 1, 297 (1984).

[21] D. J. Evans and G.P. Morriss, Phys. Rev. A 30, 1528 (1984).

[22] P. J. Hoogerbrugge and J. Koelman, Europhys. Lett. 19, 155 (1992).

[23] P. Español, Phys. Rev. E 52, 1734 (1995).

[24] J. Ramirez et al., J. Chem. Phys. 133, 154103 (2010).

[25] D. Auhl et al., J. Rheol. 52, 801 (2008). 